\title{
Differences in the expression of hepatocyte growth factor in acute and chronic bowel inflammation-Implications for diagnosis?*
}

\author{
Johanna Lönn ${ }^{1,2 \#, ~ S r a v y a ~ N a k k a ~}{ }^{1,2 \#}$, Hans Olsson ${ }^{3}$, Torbjörn Bengtsson², Sven Almer, ${ }^{4,5 \#,}$ \\ Fariba Nayeri ${ }^{1,6 \#+}$ \\ ${ }^{1}$ The Institute for Protein Environment Affinity Surveys, PEAS Institut, Linköping, Sweden \\ ${ }^{2}$ Division of Clinical Medicine, School of Health and Medical Sciences, Örebro University, Örebro, Sweden \\ ${ }^{3}$ Department of Pathology, Linköping University, Linköping, Sweden \\ ${ }^{4}$ Division of Gastroenterology and Hepatology, Karolinska Institutet, Stockholm, Sweden \\ ${ }^{5}$ Division of Gastroenterology and Hepatology, Department of Clinical and Experimental Medicine, Linköping University, Linköping, \\ Sweden \\ ${ }^{6}$ Department of Molecular and Clinical Medicine, Division of Infectious Diseases, Linköping University, Linköping, Sweden \\ Email: †Fariba.nayeri@lio.se
}

Received 1 June 2013; revised 1 July 2013; accepted 16 July 2013

Copyright (C) 2013 Johanna Lönn et al. This is an open access article distributed under the Creative Commons Attribution License, which permits unrestricted use, distribution, and reproduction in any medium, provided the original work is properly cited.

\begin{abstract}
Background: Hepatocyte growth factor (HGF) acts as an acute phase protein with regenerative properties. HGF is produced systemically and locally during inflammation but exhibits decreased binding affinity to heparan sulphate proteoglycan (HSPG)/glycosaminoglycan during chronic inflammation. We previously observed a high faecal concentration and binding affinity of HGF to HSPG during acute gastroenteritis. High faecal concentrations of calprotectin and HGF have been reported in chronic inflammatory bowel disease (IBD). Methods: Stool samples from patients with ulcerative colitis in remission $(n=11)$ or exacerbation $(n=5)$, microscopic colitis $(n=11)$, colon cancer $(n=6)$, or acute gastroenteritis caused by Clostridium difficile $(\mathrm{n}=\mathbf{2 0})$, as well as healthy controls $(\mathrm{n}=$ 7), were analysed for the presence of HGF by ELISA, surface plasmon resonance, SDS-PAGE, and Western blot. Then in two patients with ulcerative colitis exacerbation and $C$. difficile infection, the expression of HGF and calprotectin was studied in colonic biopsies. Results: The faecal concentration of HGF was significantly higher in patients with ulcerative colitis compared to the other groups. The binding affinity to dextran was lower in all groups compared to acute inflammation. HGF receptor binding was similar across
\end{abstract}

*Competing interests: The authors declare that they have no competing interests.

\#These authors contributed equally.

${ }^{\dagger}$ Corresponding author. groups. In a patient with concomitant $C$. difficile infection and distal ulcerative colitis, HGF was highly expressed in the part of the bowel unaffected by ulcerative colitis, but no expression was found at the site of chronic inflammation. In the patient with total colitis the biopsies showed low expression of HGF. The areas with chronic inflammation exhibited infiltrating calprotectin-stained neutrophils. Conclusion: HGF is produced locally during inflammation of the bowel. The HGF produced during acute inflammation or exacerbations of chronic inflammation by the unaffected area shows binding affinity to glucosaminoglycans. Measuring HGF binding in faeces and biopsies may be a tool for differentiating between acute and chronic bowel inflammation, which should be assessed thoroughly in future studies.

Keywords: Diarrhoea; Ulcerative Colitis; Dextran Sulphate; HGF; HSPG; Calprotectin

\section{INTRODUCTION}

The bowel has a wide range of functions that enable the body to metabolise food to energy in order to sustain life and protect the body from toxins and intruding microorganisms. The gastrointestinal tract is characterised by a bacterial mass in an open tube separated from a sterile environment by a few centimetres wide mucous membrane. As long as it remains intact, the immunity of the gastrointestinal system inhibits a systemic inflammatory 
response, despite being affected by numerous bacterial strains. However, in complex situations this ecosystem can be impaired, leading to inflammation and diarrhoea [1]. Thus, diarrhoea might indicate an ongoing inflammatory process in the bowel, and acute infectious gastroenteritis is a well-known cause of morbidity and death $[2,3]$.

Inflammation is usually a protective mechanism involving innate and adaptive immunity with a complex tissue response to harmful stimuli. A prolonged response of host immune cells is often caused by persistent microbial infections, which resist clearance through phagocytosis and intracellular killing. In inflammation, neutrophils are the first cells recruited from the circulation and a potent source of both anti-inflammatory and pro-inflammatory mediators and antimicrobial peptides, such as hepatocyte growth factor (HGF) and calprotectin $[4,5]$. Chronic inflammation is characterised by a shift in the type of cells present at the site of inflammation, such as a predomination of lymphocytes and macrophages [6]. Chronic inflammation may ultimately lead to tissue destruction and cancer [7].

Calprotectin is a neutrophil antimicrobial protein recently demonstrated to be valuable for ruling out serious inflammatory bowel disease (IBD) and for monitoring IBD therapy $[8,9]$. HGF is involved in biological procedures regarding development [10] and regeneration $[11,12]$. Changes in the concentration and/or structure of HGF may disturb healing or support disease development $[10,13]$. After production by mesenchymal cells, HGF is proteolytically cleaved into $\alpha$ - and $\beta$-chains and activated, and then interacts with its membrane-bound receptor, c-Met [10]. The binding affinity of HGF to heparan sulphate proteoglycan (HSPG) in the extracellular matrix or on cell membrane is crucial for inducing signal transduction [13] and subsequent elimination of HGF $[14,15]$.

Biosensor technology, such as surface plasmon resonance (SPR), allows real-time evaluation of binding affinity between ligands and receptors [16]. In SPR systems, biologically active HGF exhibits binding affinity for HSPG [17], which is decreased after the addition of sulphated oligosaccharides, such as the glycosaminoglycan/dextran sulphate [18]. In addition, biosensors coated with dextran sulphate allow the detection of HGF in cellular medium [19]. Therefore, studying the presence and quality of HGF during different inflammatory processes may be useful [20-22].

Because high amounts of HGF are produced during acute inflammation in the bowel [23], stool is an appropriate sample for investigating bowel diseases and the activation of inflammatory cells. A potential therapeutic strategy with exogenous HGF in the treatment of chronic IBD has been proposed [20,21]. Taking advantage of previous studies and observations [17,18], we investigated and compared the presence, amount, and binding affinity of HGF in faecal samples from patients with acute and chronic bowel diseases.

\section{METHODS}

\subsection{Patients}

The patients were included in a pilot study in 2003 and provided faecal samples $(n=53)$ and serum samples $(n=$ 14). Faecal $(n=7)$ and blood samples $(n=9)$ were also collected from healthy controls with no signs of diarrhoea and negative faecal cultures (Table 1). The patients with IBD were included at the Department of Gastroenterology at University Hospital in Linköping and underwent clinical, endoscopic, and pathological investigations in order to obtain the final diagnosis. None of these patients received biological therapies. In 5 of 17 cases with ulcerative colitis, the samples were collected during an acute exacerbation of disease. Two patients with ulcerative colitis exacerbation and concomitant Clostridium difficile infection underwent colonoscopy with biopsy (included in the 2003 cohort), as did a patient with colon cancer. Samples from patients with C. difficile were obtained from the Department of Microbiology in Linköping. None of the patients had known IBD. Patients with colon cancer were included at the Department of Surgery at County Hospital in Norrköping. The healthy volunteers were included when they sought vaccination before travelling abroad at the Department of

Table 1. Study subjects collected and analysed in 2003 for determination of HGF in faeces.

\begin{tabular}{|c|c|c|c|c|c|c|c|c|}
\hline \multirow{2}{*}{ Subjects (n) } & \multirow{2}{*}{ Male/Female } & \multirow{2}{*}{ Median age (range) } & \multicolumn{2}{|c|}{ Ulcerative colitis } & \multirow{2}{*}{$\begin{array}{l}\text { Microscopic } \\
\text { colitis }\end{array}$} & \multirow{2}{*}{ Culture/Toxin pos } & \multirow{2}{*}{ Duke's stage } & \multirow{2}{*}{ pos $\mathrm{FHb}$} \\
\hline & & & Active phase & Non-active & & & & \\
\hline $\operatorname{IBD}(27)$ & $10 / 17$ & $61(20-80)$ & 5 & 11 & 11 & 2 & - & nd \\
\hline C. difficile (20) & $7 / 13$ & $85(60-82)$ & & - & - & 20 & - & 10 \\
\hline Colon cancer (6) & $2 / 4$ & $75(60-82)$ & & - & - & 0 & $1-2$ & 6 \\
\hline Healthy (7) & $3 / 4$ & $40(20-60)$ & & - & - & 0 & - & 0 \\
\hline
\end{tabular}


Infectious Diseases in Linköping.

In order to investigate and compare the presence of HGF in fresh faecal samples using Western blot, faecal samples from one healthy 45 -year-old woman, a 38-yearold woman and a 15-year-old man with ulcerative colitis (exacerbation), a 54-year-old man with a urinary tract infection presenting with diarrhoea as the primary symptom (non-infectious diarrhoea), and two 78- and 80-yearold men with $C$. difficile enteritis (infectious gastroenteritis) were collected in 2012 at the Department of Infectious Diseases in Linköping.

The study was approved by the local ethical committee in Linköping. Dnr 98 - 154, 00 - 169, 02 - 094. The study subjects including the case reports have given consent.

The method used to standardise the volume of faecal samples was described previously [24]. Faecal samples were collected and stored within one hour at $-20^{\circ} \mathrm{C}$. Prior to handling the samples were thawed at room temperature and mixed by vortexing. The narrow heads of plastic syringes (Omnifix $2 \mathrm{ml}$, latex free; B. Braun Melsungen AG, Melsungen, Germany) were cut off. The plunger of the syringe was pulled out to create a small cylinder with an exact volume. The cylinder was filled and kept at $-70^{\circ} \mathrm{C}$ for 15 minutes, followed by room temperature for 1 minute to facilitate moving the faeces into the syringe. The plunger of the syringe was then pushed down to empty the cylinder into a flask $(20 \mathrm{ml}$ scintillation vial; Sarstedt AB, Landskrona, Sweden) and diluted with distilled water at a ratio of 1:6. The flask was then vortexed again. The suspension was centrifuged at 3000 $\mathrm{g}$ for 15 minutes and the supernatant transferred to new tubes (Nunc Cryo Tube; Nunc Brand Products, Soeborg, Denmark). The supernatant was stored at $-70^{\circ} \mathrm{C}$ pending analysis.

After storage and prior to analysis, the samples were thawed and centrifuged at $1000 \mathrm{~g}$ for 15 minutes. Immunoreactive HGF was determined by ELISA using commercially available kits (Quantikine HGF Immunoassay, R\&D Systems, Minneapolis, USA) according to the manufacturer's instructions. Duplicate serum and faecal samples were assessed for HGF. The minimum detection levels for the assay were $0.04 \mathrm{ng} / \mathrm{ml}$ for serum and 0.035 $\mu \mathrm{g} / \mathrm{L}$ for faeces.

\subsection{SDS PAGE and Western Blot Analysis}

Fresh unfrozen faecal samples $(15-30 \mu 1)$ were diluted in $200 \mu \mathrm{l}$ distilled water and then filtered through a 100 KDa Amicon centrifugal filter device (Millipore, Solna, Sweden). Filtrate obtained upon centrifugation was mixed with Laemmli sample buffer $(1 \mathrm{x})$ containing 2mercaptoethanol $\left(98^{\circ} \mathrm{C}\right.$ for $\left.5 \mathrm{~min}\right)$ in a $1: 1$ ratio and then heated to $95^{\circ} \mathrm{C}$ to denature the proteins. The proteins were separated using standard sodium dodecyl sulfate- polyacrylamide gel electrophoresis (SDS-PAGE) and electrotransferred to a polyvinylidene difluoride membrane. Non-specific binding was blocked by incubating the membranes with $5 \%$ dry milk in Tris-buffer saline (TBS; $25 \mathrm{mM}$ Tris base, $150 \mathrm{mM} \mathrm{NaCl}, 2 \mathrm{mM} \mathrm{KCl} \mathrm{pH}$ 7.4 , and $0.1 \%$ Tween-20) for $1 \mathrm{~h}$ at room temperature prior to incubation with a goat polyclonal anti-HGF antibody (1:1000; AF-294-NA) for $1 \mathrm{~h}$ at room temperature. The membranes were then incubated with a polyclonal HRP-conjugated donkey anti-goat antibody (1:1000; HAF 109). Recombinant HGF (294-HA; R\&D systems, Minneapolis, MN, USA) was used as a positive control.

\subsection{SPR and Ligand Immobilisation}

SPR was performed at $760 \mathrm{~nm}$ in a fully automatic Biacore 2000 instrument (GE Healthcare, Uppsala, Sweden) equipped with four flow cells. Faecal samples were thawed and diluted with phosphate buffer solution $(\mathrm{pH}$ 7.4, Apoteket AB, Umeå, Sweden) at a ratio of 1:1. To avoid the effects of digestive and bacterial enzymes, a protease inhibitor mix $(1 \%-5 \%)$ containing 4 -(2-aminoethyl) benzenesulfonyl fluoride (AEBSF), pepstatin A, E-64, bestatin, leupeptin, and aprotinin (but no metal chelators) (Sigma Aldrich), with specific inhibition of serine, cysteine, aspartic proteases, and aminopeptidases, was added to the thawed samples at room temperature within the 30 minutes prior to analysis. The monoclonal anti-HGF antibody (500 $\mu \mathrm{g} / \mathrm{ml}$, R\&D Systems) was diluted 1:10, and the recombinant c-Met proto-oncogene receptor $(100 \mu \mathrm{g} / \mathrm{ml}, \mathrm{R} \& \mathrm{D}$ Systems $) 1: 5$, in $10 \mathrm{mM}$ acetate buffer ( $\mathrm{pH} 4.5)$ and immobilised on a CM5 chip. The last flow cell was used to monitor the binding of HGF to dextran. This flow cell was treated the same way for the immobilisation procedure, but the ligand immobilisation step was omitted. The contact time for analysis of faeces was 3 minutes, and the surfaces were washed with 1-min injections of $5 \mathrm{mM}$ glycine buffer ( $\mathrm{pH} 2.0$ ) containing 1 $\mathrm{M} \mathrm{NaCl}$. Serum was diluted in PBS in a 1:20 ratio and run for 3 minutes. The surfaces were washed with 1-min injections of $5 \mathrm{mM}$ glycine buffer (pH 2.0) containing 1 $\mathrm{M} \mathrm{NaCl}$, followed by a 1-min injection of borate $(\mathrm{pH} 8.5$; GE Healthcare). Positive and negative controls were included at the beginning and end of each run to confirm the reliability of the surfaces. The SPR data were obtained as response units (RUs) [25].

\subsection{Immunohistochemical Staining}

The immunohistochemical analyses were performed on formalin-fixed and paraffin-embedded bowel biopsies using a biotin-free immunoperoxidase method (IntelliPath immunostainer, Biocare, Pike Lane Concord, CA, USA). Fresh $4-\mu \mathrm{m}$ tissue sections were cut from the paraffin blocks and heated at $60^{\circ} \mathrm{C}$ for 2 hours. The sec- 
tions were then deparaffinised, rehydrated, and pre-treated with heat-induced epitope retrieval in a Diva Decloaker buffer ( $\mathrm{pH}$ 6.0; Biocare medical) in an automatic pressure cooker for $30 \mathrm{sec}$ at full pressure to improve staining. The primary antibodies were monoclonal antiHGF (Sigma H1896, clone 24612.111; diluted 1:25) and polyclonal anti-calprotectin (Anti-S100A8, Sigma-Aldrich; diluted 1:5000). Mouse anti-IgG1/IgM (SigmaAldrich) was used as a negative control. A polymer detection kit containing diaminobenzidine tetrahydrochloride (MACH 4 Universal HRP-Polymer Kit with DAB, Biocare Medical) was used as the chromogen to produce the reaction product, followed by haematoxylin counterstaining, dehydration, and coverslip mounting. The staining was observed under a microscope (Nikon Eclipse E600) with attached camera (Nikon Digital Sight DS-U2; Nikon, Solna, Sweden).

\subsection{Statistical Analysis}

The data were not normally distributed. Therefore, the Mann-Whitney U-test was used in Graph Pad Prism version 5. When comparing more than two groups, the Kruskal-Wallis test was used first. The median and interquartile ranges (IQR) are presented and $P<0.05$ was considered significant.

\section{RESULTS}

\subsection{Expression of Faecal HGF in SDS-PAGE and Western Blot}

SDS-PAGE and immunoblotting analysis was performed to distinguish HGF during infection and ulcerative colitis, from that of healthy controls. Faecal samples from patients with C. difficile infection resulted in bands at 64 $\mathrm{KDa}$, corresponding to the $\alpha$-chain of HGF, and at 34 $\mathrm{KDa}$ and $37 \mathrm{KDa}$, corresponding to the $\beta$-chain (Figure
1). Weak bands of approximately $64 \mathrm{KDa}$ were detected for the faecal sample from a patient with a urinary tract infection who presented with culture-negative diarrhoea (patient with diarrhoea but no infection) and the sample from a healthy individual. For the ulcerative colitis samples, several precursor bands were obtained between 68 and $95 \mathrm{KDa}$, as well as bands at $34-37 \mathrm{KDa}$. These bands were confirmed by Western blot analysis (Figure 1, right panel). No bands were observed on the Western blot for samples from healthy controls or the patient with diarrhoea but no infection (data not shown).

\subsection{Binding Affinity and Concentration of HGF Measured by SPR and ELISA}

The binding affinity to dextran, monoclonal anti-HGF antibody, and HGF receptor (c-Met) and the concentra-

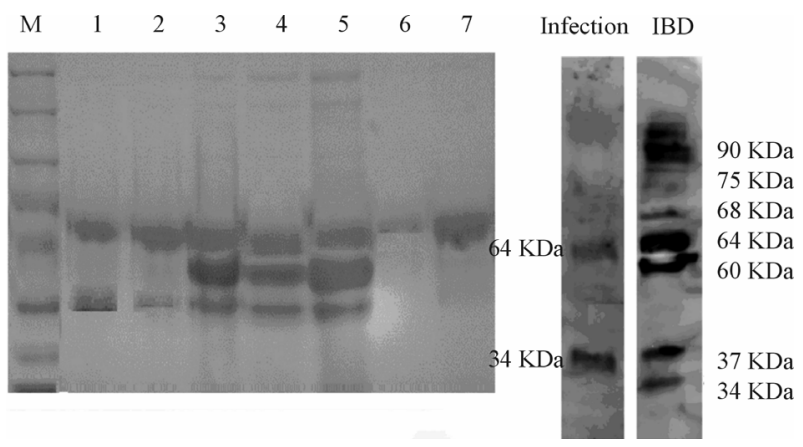

Figure 1. SDS PAGE analysis of faecal samples. Wells 1, 2: $C$. difficile infection; wells 3 - 5: ulcerative colitis ( 3 and 5 are the same sample from the patient with acute exacerbation); well 6: diarrhoea during the course of urinary tract infection; well 7: healthy control. Patients with diarrhoea due to infection or ulcerative colitis had bands at 64 and $34 \mathrm{KDa}$, i.e. the $\alpha$ - and $\beta$-chains of HGF, respectively; this was confirmed by Western blot (right panel). Patients with ulcerative colitis also had bands at $90 \mathrm{KDa}$, corresponding to precursor HGF on Western blot.

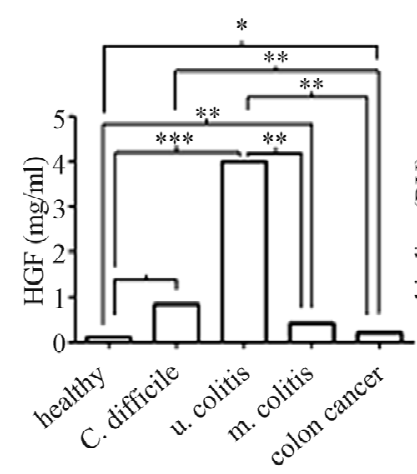

(a)

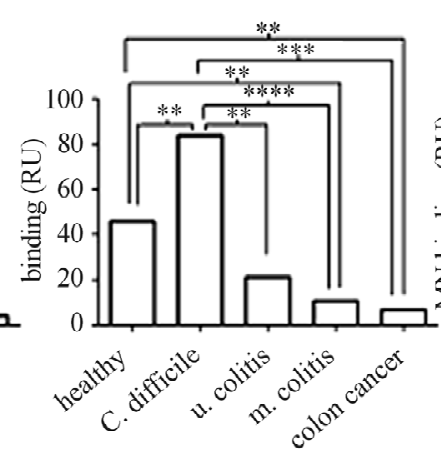

(b)

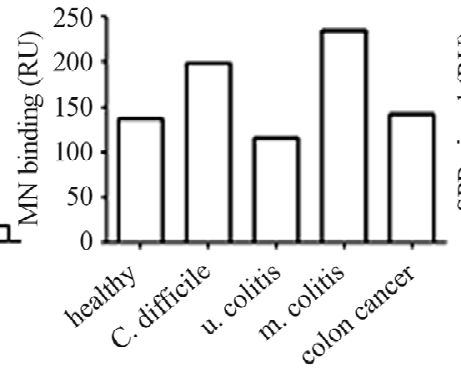

(c)

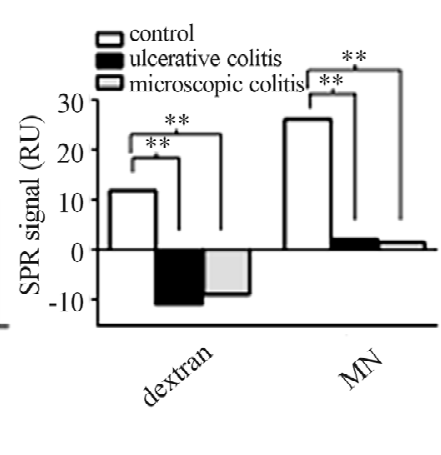

(d)

Figure 2. Ligand binding affinity and concentration of HGF in faeces and serum. (a) Median faecal concentration of HGF (ng/ml) measured by ELISA. (b) Median binding affinity to dextran and (c) monoclonal anti-HGF antibody (MN) analysed by surface plasmon resonance (SPR; RU). (d) Median binding affinity to dextran and MN was also measured in serum from patients with ulcerative colitis (black column) and microscopic colitis (grey) compared to healthy blood donors (white column). ${ }^{*} P<0.05,{ }^{* *} P<0.01,{ }^{* * *} P<$ $0.001,{ }^{* * * *} P<0.0001$ according to Kruskal-Wallis and Mann-Whitney U-test. 
tion of HGF were analysed by SPR and ELISA. All groups had a significantly increased concentration of HGF in the faecal samples compared to healthy controls, and patients with ulcerative colitis $(n=16)$ had significantly higher concentrations of HGF compared to all of the other groups except infection (Figure 2(a)). However, the binding affinity to dextran was lower in all groups except patients with ulcerous colitis $(\mathrm{n}=16)$ compared to healthy controls. All groups exhibited significantly lower dextran binding compared to patients with gastroenteritis caused by C. difficile (Figure 2(b)). Because of few cases, no comparison was done between the patients with exacerbation $(\mathrm{n}=5)$ to the other patients with ulcerative colitis $(n=11)$.

In serum from patients with ulcerative $(n=4)$ or microscopic $(n=10)$ colitis, lower binding affinity to both dextran and monoclonal anti-HGF was observed compared to healthy blood donors $(\mathrm{n}=9)$ (Figure 2(d)). The binding affinity of HGF to c-Met did not significantly differ between the groups.

\subsection{Case Report 1}

A woman born in 1961 with a medical history of ulcerative colitis since 1994 and no family history of ulcerative colitis was admitted for having diarrhoea for 3 weeks, with fever and bloody stool for 3 days. The patient was taking basalazide and lansoprazole. Computed tomography revealed distal colitis without signs of obstruction or dilatation. Stool cultures revealed growth of toxin Aproducing $C$. difficile. She received oral metronidazole $(500 \mathrm{mg} \times 3)$ from the second day of hospitalisation without dramatic relief of symptoms. However, after adding oral prednisolone (30 mg daily) and rectal application of mesalazine the following day, her symptoms improved and she underwent sigmoidoscopy on the fourth day of hospitalisation. High grade inflammation (grade II - III) was observed with a sharp limit $25 \mathrm{~cm}$ from the anal verge (Figure 3), and biopsies were taken distal and proximal to this border. Histopathology showed slight inflammation in the proximal biopsies and moderate chronic active inflammation and the presence of cryptitis in the distal biopsies.

High levels of HGF with high affinity for dextran were detected in the faecal samples (Table 2). Western blot revealed two bands at 65 and $35 \mathrm{KDa}$, reflecting the $\alpha$ and $\beta$-chains, respectively (Figure 4). Immunohistochemistry revealed higher expression of HGF (Figure 5) and c-Met receptor (data not shown) in biopsies taken proximally from unaffected area compared to distal biopsies taken from the area of chronic inflammation. The distal biopsy exhibited more infiltrating calprotectinstained neutrophils compared to the biopsy taken proximally (Figure 5).
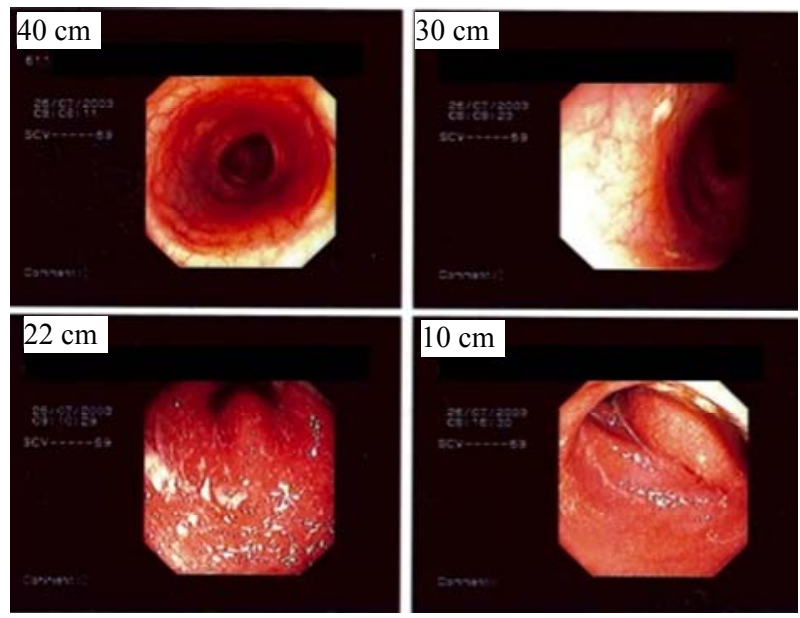

Figure 3. Endoscopic images of distal ulcerative colitis and Clostridium difficile infection in a 42-year-old woman. The images were taken $40 \mathrm{~cm}, 30 \mathrm{~cm}, 22 \mathrm{~cm}$, and $10 \mathrm{~cm}$ from the anal verge.

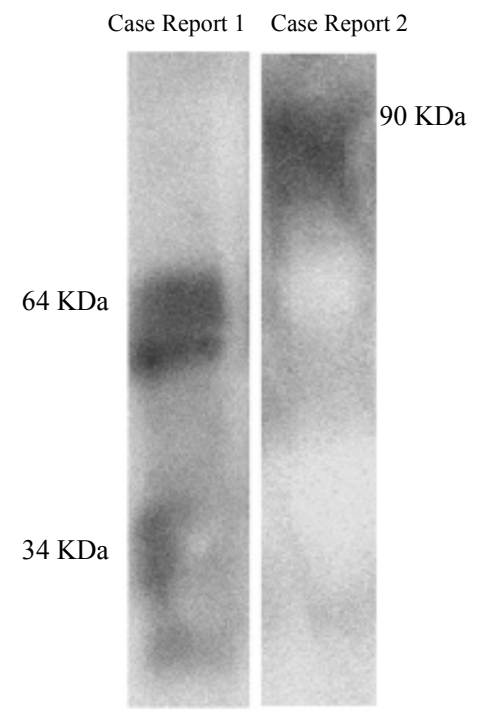

Figure 4. Western blot analysis of HGF in case reports 1 and 2. Faecal samples were diluted in 1:1 PBS, centrifuged, and subjected to reduced SDS-PAGE analysis. Separated proteins were transferred to a PVDF membrane and detected with goat polyclonal anti-HGF primary antibody (1:1000; AF-294-NA) binding to a secondary polyclonal HRPconjugated donkey anti-goat antibody (1:1000; HAF 109).

We also observed that the level of C-reactive protein (CRP), a marker of inflammation, decreased after corticosteroid administration and continued to decrease to 26 $\mathrm{mg} / \mathrm{L}$ on 28 July. Therefore, despite the presence of $C$. difficile (both growth in faecal culture and toxin), the patient's symptoms may depend on an exacerbation of 
Table 2. Faecal and serum samples from a patient with $C$. difficile infection and distal ulcerative colitis.

\begin{tabular}{cccccc}
\hline Date July 2003 & $\begin{array}{c}\text { Faecal HGF } \\
\text { ng/ml }\end{array}$ & $\begin{array}{c}\text { Serum CRP } \\
\mathrm{mg} / \mathrm{L}\end{array}$ & \multicolumn{2}{c}{ SPR (Biacore ${ }^{\circledR}$ ) binding to epitopes (RU) (Faeces) } \\
\hline $21^{\text {st }}$ (admission) & 6.06 & 222 & 47 & 27 & 18 \\
$22^{\text {th }}$ (antibiotics) & 61.6 & 211 & 447 & 356 & 153 \\
$24^{\text {th }}$ (prednisolone) & 6.36 & 206 & 203 & 262 & 44 \\
$25^{\text {th }}$ (endoscopy) & nd & 186 & 175 & 180 & 49 \\
\hline
\end{tabular}

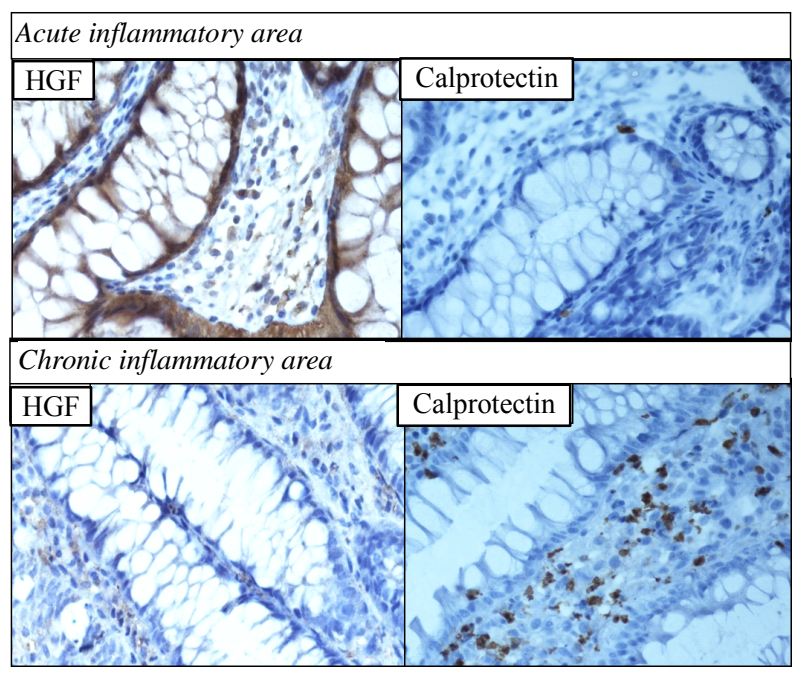

Figure 5. Immunohistochemical staining of hepatocyte growth factor (HGF) and calprotectin in case report 1. Biopsies were taken from the proximal (upper panel) unaffected/acute inflammation area and the distal (lower panel) affected area with ulcerative colitis (magnification $40 \times$ ). In contrast to the lack of HGF expression in the chronic inflammation sample, which exhibited increased calprotectin-stained neutrophils, HGF was expressed to a high degree in the unaffected area, which exhibited low calprotectin expression. Taken together with the presence of high amounts of HGF with high affinity to dextran in faecal samples collected at the same time as endoscopy (Table 2), this result may indicate autocrine production of HGF by the unaffected area.

ulcerative colitis.

\subsection{Case Report 2}

A 40-year-old man suffering from narcotic and alcohol overconsumption, chronic hepatitis $\mathrm{C}$ infection, cirrhosis, and ulcerative colitis was admitted to intensive care unit in May 2003 with gastrointestinal bleeding, diarrhoea, and fever. X-rays showed total colitis. The CRP level was $<10 \mathrm{mg} / \mathrm{ml}$. The patient was initially treated with intravenous cefotaxime and oral metronidazole. Stool cultures revealed growth of $C$. difficile. The patient underwent colonoscopy on the fourth day of hospitalisation (Figure 6). Histopathology revealed severe chronic inflammation and moderate active colitis resembling

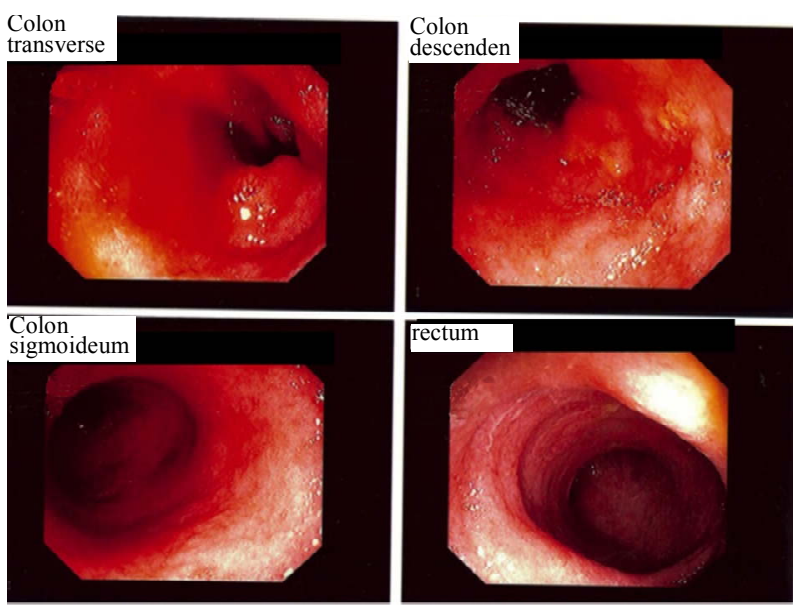

Figure 6. Colonoscopy images from a 40-year-old man with total ulcerative colitis. Severe inflammation was found in all parts of the colon.

ulcerative colitis in the whole colon. Immunohistochemistry revealed slight expression of HGF and an increased number of calprotectin-stained neutrophils were observed (Figure 7).

Western blot analysis of faecal samples showed only one band corresponding to the HGF precursor (90 KDa) (Figure 8). The concentration of HGF in faeces was 11 $\mathrm{ng} / \mathrm{ml}$. Binding affinity of faecal HGF to glucosaminoglycans and monoclonal anti-HGF antibody in SPR was low ( $<5.0$ RU). He was admitted several times with similar symptoms and ultimately died in January 2004.

\subsection{Case Report 3}

Colonic biopsies were taken from the intact area and tumour area of an 81-year-old man with colon cancer (Duke's stage 1) and studied for the expression of HGF and calprotectin. Low expression of HGF and calprotectin was observed in the tumour area, whereas no staining was observed in the biopsy taken from the intact area (Figure 8).

\section{DISCUSSION}

Here, we have studied samples from patients with bowel disorders and healthy and shown that acute inflammation 
HGF

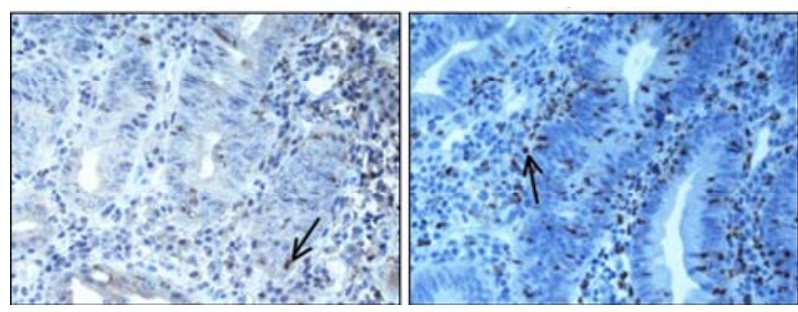

Figure 7. Immunohistochemistry of hepatocyte growth factor (HGF) (left) and calprotectin (right) in case report 2. The biopsy was taken from a 40-year-old man with total ulcerative colitis (magnification $40 \times$ ).

Intact area in patient with colon cancer
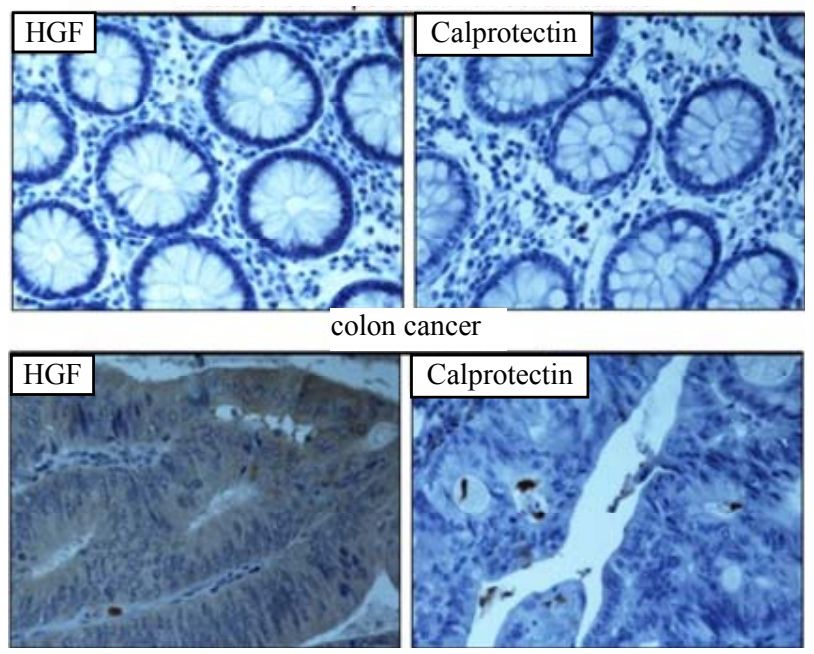

Figure 8. Immunohistochemistry of HGF (left) and calprotectin (right) expression in colon cancer. Bowel biopsies were taken from the intact area (upper panel) and tumour area (lower panel) of an 81-year-old man with colon cancer (magnification $40 \times$ ).

can be discriminated from the chronic inflammatory disorders based on the presence and quality of HGF produced locally at the site of injury in the bowel. The expression of HGF and calprotectin in biopsies inversely correlated with each other.

The receptor for HGF is c-Met [10], but for the induction of cellular responses, the interaction between HGF and the co-receptor HSPG is essential. HSPG binds and facilitates the cytokine-receptor interaction, as well as the conversion of promitogen HGF to the mature twochain form [26]. Despite being present in high concentrations, HGF may appear in a form with reduced biological activity, characterised by reduced binding affinity to HSPG and dextran sulphate and decreased regeneration in a model of cell injury (i.e., mouse skin epithelial cells, CCL-53.1) [27,28]. HSPG and dextran sulphate are both glucosaminoglycans and bind to the same epitope on the HGF molecule [18].

Two decades ago, profound studies were published about the physiological aspects of HGF in health and development $[29,30]$. In recent years, however, several groups have focused on assessing HGF during inflammation and cancer [10]. We previously showed that patients with chronic/acute skin ulcers [17,18], chronic renal failure [31], ischemic heart disease [32], and periodontitis [33] express a high concentration of HGF but a decreased binding affinity of HGF to glucosaminoglycans. In the present study, we observed a higher concentration of $\mathrm{HGF}$ in both acute inflammation (i.e., C. difficile infection) and chronic inflammation (i.e., ulcerative and microscopic colitis and cancer) compared to healthy controls. However, in contrast to chronic inflammation, HGF produced during the acute inflammation in the bowel had high affinity to antibodies as well as glucosaminoglycans. Faecal HGF exhibited two bands on Western blot, belonging to the $\alpha$ - and $\beta$-chain, which is in agreement with our previous observations [25]. However, binding to the HGF receptor (c-Met) did not differ between healthy, acute inflammation, and chronic inflammation. Buchstein et al. demonstrated an inability of HGF to induce c-Met signalling in secretions from chronic non-healing skin ulcers [34]. During chronic inflammation, HGF may have the ability to bind to c-Met but be unable to induce cell signalling due to deficient ligation to the co-receptor HSPG.

HGF is produced rapidly after injury and ongoing damage, acting as an acute phase protein, and our present data is in agreement with our previous observations that HGF may be a more sensitive marker for monitoring the effect of treatment than CRP [35] (Table 2). Furthermore, knock-out mice lacking the HGF gene do not survive [36], and HGF administration has been used to inhibit and treat several injuries, thus acting as a potent growth factor [10]. Consequently, specific aspects and properties of HGF need to be observed to elucidate its role as a diagnostic and therapeutic agent.

In one of our two patients with concomitant $C$. difficile infection and ulcerative colitis, biopsy studies revealed two distinct but adjacent areas of colonic inflammation with different expression levels of HGF and calprotectin. Low staining for HGF was observed at sites of chronic inflammation, whereas opposite findings were observed in unaffected areas for ulcerative colitis/with acute inflammation. HGF was expressed on epithelial cells in unaffected areas of the bowel instead of in the mesenchymal cells where HGF is produced. Liu et al. [15] described the presence of at least two binding sites for HGF on liver cell surfaces: the heparin-washable site (HSPG) and the heparin-resistant and acid-washable binding site (c-Met receptor) considered to have higher affinity for HGF. Epithelial cells in the bowel are also supposed to contain these two binding sites. Though specimens from colon biopsies were formalin-fixed and 
paraffin-embedded prior to staining, we assume that the expression of HGF on bowel epithelial cells in the unaffected areas indicates the binding of HGF to HSPG. We previously observed that the addition of dextran sulphate and heparin to samples containing biologically active HGF (such as faeces from acute gastroenteritis patients) resulted in significantly reduced binding to the channel immobilised with HSPG in the SPR system [25]. This result might indicate that the addition of glucosaminoglycans, such as heparin and dextran sulphate, to samples containing HGF inhibits the interaction between HGF and the heparin-washable binding sites, HSPG. On the other hand, HGF staining on bowel epithelial cells in acute inflammation samples might indicate that such binding is decreased during chronic inflammation. In addition, the HGF concentration was high in faecal samples from this patient (Table 2), and HGF exhibited a binding affinity for dextran in SPR. The case report 1 might indicate the paracrine production of biologically active HGF from mesenchymal cells in the healthy parts of the colon that interacts with neighbouring epithelial cells to replace the injured cells and induce healing [10]. In case report 2 with total colitis the expression of HGF in biopsies and the binding affinity to glucosaminoglycans in SPR was low. We found a high number of calprotectin-expressing cells in areas of chronic inflammation. Similar observations regarding calprotectin expression were reported previously for IBD [37].

The present study has limitations. The study material is small, the patients with ulcerative colitis were in different stages of disease activity and C. difficile was the only infectious agent assessed as a cause of acute inflammation in the bowel. However, the results regarding HGF and calprotectin are in agreement with previous observations. We think that further studies investigating the correlation between HGF and calprotectin may be of great value for the differential diagnosis of acute and chronic inflammatory disease in the bowel upon admission.

\section{CONCLUSION}

The expression and functional capacity of HGF differ significantly between acute and chronic bowel inflammation. These findings may be helpful in the development of diagnostic and therapeutic strategies for various forms of IBD.

\section{ACKNOWLEDGEMENTS}

We are grateful to Tayeb Nayeri for establishing the method for analysing HGF in faeces using SPR and the observation of a correlation between HSPG-binding and the biological activity of HGF. We are grateful to Lise-Lott Lindvall for nursing assistance and to Birgitta Frånlund for immunohistochemical assays.
This study was supported by the Medical Research Council of Southeast Sweden (FORSS), Linköping University, and the County Council of Östergötland (ALF-grants).

\section{REFERENCES}

[1] Juckett G. and Trivedi, R. (2011) Evaluation of chronic diarrhea. American Family Physician, 84, 1119-1126.

[2] Pardi, D.S. (2012) Miscellaneous colitides. Current Opinion in Gastroenterology, 28, 76-81. doi:10.1097/MOG.0b013e32834dda3b

[3] Wilkins, T., Jarvis, K. and Patel, J. (2011) Diagnosis and management of Crohn's disease. American Family Physician, 84, 1365-1375.

[4] Grenier, A., Chollet-Martin, S., Crestani, B., Delarche, C., El Benna, J., Boutten, A., Andrieu, V., Durand, G., Gougerot-Pocidalo, M.A., Aubier, M. and Dehoux, M. (2002) Presence of a mobilizable intracellular pool of hepatocyte growth factor in human polymorphonuclear neutrophils. Blood, 99, 2997-3004. doi:10.1182/blood.V99.8.2997

[5] Striz, I. and Trebichavsky, I. (2004) Calprotectin-A pleiotropic molecule in acute and chronic inflammation. Physiological Research, 53, 245-53.

[6] Kumar, R.K. and Wakefield, D. (2001) Inflammation: Chronic. John Wiley \& Sons, Ltd., Hoboken.

[7] Cromer, W.E., Mathis, J.M., Granger, D.N., Chaitanya, G.V. and Alexander, J.S. (2011) Role of the endothelium in inflammatory bowel diseases. World Journal of Gastroenterology, 17, 578-593. doi:10.3748/wjg.v17.i5.578

[8] Kostakis, I.D., Cholidou, K.G., Vaiopoulos, A.G., Vlachos, I.S., Perrea, D. and Vaos, G. (2012) Fecal calprotectin in pediatric inflammatory bowel disease: A systematic review. Digestive Diseases and Sciences, 58, 309319. doi:10.1007/s10620-012-2347-5

[9] Chen, C.C., Huang, J.L., Chang, C.J. and Kong, M.S. (2012) Fecal calprotectin as a correlative marker in clinical severity of infectious diarrhea and usefulness in evaluating bacterial or viral pathogens in children. Journal of Pediatric Gastroenterology and Nutrition, 55, 541-547. doi:10.1097/MPG.0b013e318262a718

[10] Nakamura, T., Sakai, K. and Matsumoto, K. (2011) Hepatocyte growth factor twenty years on: Much more than a growth factor. Journal of Gastroenterology and Hepatology, 26, 188-202. doi:10.1111/j.1440-1746.2010.06549.x

[11] Doeppner, T.R., Kaltwasser, B., ElAli, A., Zechariah, A., Hermann, D.M. and Bahr, M. (2011) Acute hepatocyte growth factor treatment induces long-term neuroprotection and stroke recovery via mechanisms involving neural precursor cell proliferation and differentiation. Journal of Cerebral Blood Flow \& Metabolism, 31, 1251-62. doi:10.1038/jcbfm.2010.211

[12] Nayeri, F., Strömberg, T., Larsson, M., Brudin, L., Söderström, C. and Forsberg, P. (2002) Hepatocyte growth factor may accelerate healing in chronic leg ulcers: A pilot study. Journal of Dermatological Treatment, 13, 81-86. doi:10.1080/095466302317584449

[13] Derksen, P.W., Keehnen, R.M., Evers, L.M., van Oers, 
M.H., Spaargaren, M. and Pals, S.T. (2002) Cell surface proteoglycan syndecan-1 mediates hepatocyte growth factor binding and promotes Met signaling in multiple myeloma. Blood, 99, 1405-1410.

doi:10.1182/blood.V99.4.1405

[14] Hartmann, G., Prospero, T., Brinkmann, V., Ozcelik, O., Winter, G., Hepple, J., Batley, S., Bladt, F., Sachs, M., Birchmeier, C., Birchmeier, W. and Gherardi, E. (1998) Engineered mutants of $\mathrm{HGF} / \mathrm{SF}$ with reduced binding to heparan sulphate proteoglycans, decreased clearance and enhanced activity in vivo. Current Biology, 8, 125-134. doi:10.1016/S0960-9822(98)70059-4

[15] Liu, K.X., Kato, Y., Kato, M., Kaku, T.I., Nakamura, T. and Sugiyama, Y. (1997) Existence of two nonlinear elimination mechanisms for hepatocyte growth factor in rats. American Journal of Physiology, 273, E891-E897.

[16] Liedberg, B., Nylander, C. and Lundström, I. (1995) Biosensing with surface plasmon resonance-How it all started. Biosensors and Bioelectronics, 10, i-ix. doi:10.1016/0956-5663(95)96965-2

[17] Nayeri, F., Xu, J., Abdiu, A., Nayeri, T., Aili, D., Liedberg, B. and Carlsson, U. (2006) Autocrine production of biologically active hepatocyte growth factor (HGF) by injured human skin. Journal of Dermatological Science, 43, 49-56. doi:10.1016/j.jdermsci.2006.03.004

[18] Nayeri, F., Nayeri, T., Aili, D., Brudin, L. and Liedberg, B. (2008) Clinical impact of real-time evaluation of the biological activity and degradation of hepatocyte growth factor. Growth Factors, 26, 163-171. doi: $10.1080 / 08977190802128083$

[19] Berger, M., Welle, A., Gottwald, E., Rapp, M. and Lange, K. (2010) Biosensors coated with sulfated polysaccharides for the detection of hepatocyte growth factor/scatter factor in cell culture medium. Biosensors and Bioelectronics, 26, 1706-1709. doi:10.1016/j.bios.2010.07.065

[20] Yamaji, N., Ido, A., Moriuchi, A., Numata, M., Setoyama, H., Tamai, T., Funakawa, K., Fujita, H., Sakiyama, T., Uto, H., Oketani, M. and Tsubouchi, H. (2011) Hepatocyte growth factor ameliorates mucosal injuries leading to inhibition of colon cancer development in mice. Oncology Reports, 26, 335-341.

[21] Linares, P.M. and Gisbert, J.P. (2011) Role of growth factors in the development of lymphangiogenesis driven by inflammatory bowel disease: A review. Inflammatory Bowel Diseases, 17, 1814-1821. doi:10.1002/ibd.21554

[22] Cammarota, R., Bertolini, V., Pennesi, G., Bucci, E.O., Gottardi, O., Garlanda, C., Laghi, L., Barberis, M.C., Sessa, F., Noonan, D.M. and Albini, A. (2010) The tumor microenvironment of colorectal cancer: Stromal TLR-4 expression as a potential prognostic marker. Journal of Translational Medicine, 8, 112. doi:10.1186/1479-5876-8-112

[23] Nayeri, F., Almer, S., Brudin, L., et al. (2003) High heaptocyte growth factor levels in faeces during acute infectious gastroenteritis. Scandinavian Journal of Infectious Diseases, 35, 858-862. doi:10.1080/00365540310016484

[24] Nayeri, F., Nilsson, I., Brudin, L. and Almer, S. (2004) Stability of faecal hepatocyte growth factor determination. Scandinavian Journal of Clinical \& Laboratory Investi- gation, 64, 589-597. doi:10.1080/00365510410002850

[25] Nayeri, F., Aili, D., Nayeri, T., Xu, J., Almer, S., Lundström, I., Akerlind, B. and Liedberg, B. (2005) Hepatocyte growth factor (HGF) in fecal samples: Rapid detection by surface plasmon resonance. BMC Gastroenterology, 5, 13. doi:10.1186/1471-230X-5-13

[26] Rubin, J.S., Day, R.M., Breckenridge, D., Atabey, N., Taylor, W.G., Stahl, S.J., Wingfield, P.T., Kaufman, J.D., Schwall, R. and Bottaro, D.P. (2001) Dissociation of heparan sulfate and receptor binding domains of hepatocyte growth factor reveals that heparan sulfate-c-met interaction facilitates signaling. The Journal of Biological Chemistry, 276, 32977-32983. doi:10.1074/jbc.M105486200

[27] Lönn, J., Almroth, G., Brudin, L. and Nayeri, F. (2012) An Antithrombin III product containing biologically active hepatocyte growth factor may be beneficial in deep ulcer infections. Cytokine, 60, 478-486. doi:10.1016/j.cyto.2012.05.023

[28] Nayeri, F., Holmgren-Pettersson, K., Perskvist, N., Forsberg, P., Peterson, C. and Sundqvist, T. (2007) An in vitro model for assessment of the biological activity of hepatocyte growth factor. Growth Factors, 25, 33-40. doi:10.1080/08977190600997200

[29] Wang, X., DeFrances, M.C., Dai, Y., Pediaditakis, P., Johnson, C., Bell, A., Michalopoulos, G.K. and Zarnegar, R. (2002) A mechanism of cell survival: Sequestration of Fas by the HGF receptor Met. Molecular Cell, 9, 411-421. doi:10.1016/S1097-2765(02)00439-2

[30] Defrances, M.C., Wolf, H.K., Michalopoulos, G.K. and Zarnegar, R. (1992) The presence of hepatocyte growth factor in the developing rat. Development, 116, 387-395.

[31] Lönn, J., Shahzad, F., Uhlin, F., Bengtsson, T., Almroth, G. and Nayeri, F. (2012) High concentration but low biological activity of hepatocyte growth factor in patients with chronic renal failure. Advances in Bioscience and Biotechnology, 3, 516-523. doi:10.4236/abb.2012.324068

[32] Lönn, J., Starkhammar Johansson, C., Kälvegren, H., Brudin, L., Skoglund, C., Garvin, P., Särndahl, E., Ravald, N., Richter, A., Bengtsson, T. and Nayeri, F. (2012) Hepatocyte growth factor in patients with coronary artery disease and its relation to periodontal condition. Results in Immunology, 2, 7-12. doi:10.1016/j.rinim.2011.12.002

[33] Lönn, J., Starkhammar Johansson, C., Nakka, S., Palm, E., Bengtsson, T., Nayeri, F. and Ravald, N. (2013) High concentration but low biological activity of hepatocyte growth factor in patients with periodontitis. Journal of Periodontology. doi:10.1902/jop.2013.130003

[34] Buchstein, N., Hoffmann, D., Smola, H., Lang, S., Paulsson, M., Niemann, C., Krieg, T. and Eming, S.A. (2009) Alternative proteolytic processing of hepatocyte growth factor during wound repair. American Journal of Pathology, 174, 2116-2128. doi:10.2353/ajpath.2009.080597

[35] Abednazari, H., Xu, J., Millinger, E., Brudin, L., Forsberg, P. and Nayeri, F. (2006) Hepatocyte growth factor is a better indicator of therapeutic response than C-reactive protein within the first day of treatment in pneumonia. Chemotherapy, 52, 260-263.

doi: $10.1159 / 000094868$ 
[36] Uehara, Y., Mori, C., Noda, T., Shiota, K. and Kitamura, N. (2000) Rescue of embryonic lethality in hepatocyte growth factor/scatter factor knockout mice. Genesis, 27, 99-103.

doi:10.1002/1526-968X(200007)27:3<99::AID-GENE20 $>3.0 . \mathrm{CO} ; 2-\mathrm{Y}$
[37] Manolakis, A.C., Kapsoritakis, A.N., Tiaka, E.K. and Potamianos, S.P. (2011) Calprotectin, calgranulin C, and other members of the s100 protein family in inflammatory bowel disease. Digestive Diseases and Sciences, 56, 1601-1611. doi:10.1007/s10620-010-1494-9 